\title{
The Use of Orbital Angular Momentum of Light Beams for Optical Data Storage
}

\author{
Robbert Jan Voogd, Mandeep Singh, Joseph J.M. Braat* \\ Optics Research Group, TNW/IST, TU Delft, Lorentzweg 1, 2628 CJ Delft, The Netherlands
}

\begin{abstract}
We present a method to optically store multiple information in one location by having angular momentum imparted to the scanning beam by optical phase objects that make up the information areas on a surface. We show that the light beam thus perturbed carries an optical vortex, the rotation of which can be detected in the near-field focal region as a function of the longitudinal distance either side of the beam waist at $z_{0}$. The spatial variation of the rotation of the $E$ field and hence the intensity is dependent upon the phase depth (typically in the range $\pi / 2$ to $2 \pi$ ) introduced by quadrant staircase-type structures which approximate well to helical phase defects. We propose that using suitable detection schemes, this spatial variation (rotation) of intensity profiles can be used to extract multiple bits of information from the different quadrant staircase structures of varying phase depths.
\end{abstract}

Keywords: Orbital angular momentum, optical vortices, multiplexing, optical data storage

\section{INTRODUCTION}

In the field of optical recording, the increase in spatial density has primarily been achieved by reducing the quantity $\lambda / N A$, the diffraction unit, where $\lambda$ is the wavelength, and $N A$ the numerical aperture. To a lesser extent, spatial density increase has been realized by a further reduction of fabrication and system tolerances and by exploiting the margins in the signal to noise ratio, e.g. by two- or three-level intensity recording. A further reduction of the diffraction unit along the wavelength path is unlikely because of the absence of cheap UV sources. The $N A$ increase beyond the unit value requires immersion read-out or optical tunneling. Both techniques are not practical, the first one because of handling problems, the second because of system and medium robustness. From the very beginning, together with removability, system robustness has been the most attractive feature of optical recording for the customer and any compromise in this direction would severely limit the use of optical discs for the cheap storage of massive amounts of information.

A possible road towards higher information density and data retrieval rate in optical storage is the recording of more than one bit per storage location (multiplexing). In this paper we analyze the introduction of angular momentum by specially designed optical effects on an optical disc and the read-out of these multiplexed effects by a quadrant-type detector. We think that conveying angular momentum from the optical effect to the detector arrangement is a process that is robust because of the basic conservation law for angular momentum $;{ }^{1}$ common sources of noise and defects have a very limited influence on the angular momentum detection method. It can be expected that both the sign of the angular momentum and several stages in its amplitude can be adequately detected.

\section{THEORY AND MODELING}

The angular momentum of a (focused) beam of light comprises an intrinsic part due to the photon spin (units $\pm \hbar$ or $\pm h / 2 \pi$ per photon) and a part that can be associated with the geometrical structure of a light beam. ${ }^{1,2}$ Beams carrying net spin momentum have to be non plane-polarized and the rotation sense of the generally elliptically polarized light determines the sign of the spin angular momentum. Geometrically introduced orbital angular momentum is encountered when a light beam has passed through e.g. a discontinuous phase plate whose transmission function is given, in cylindrical coordinates, by ${ }^{3}$

\footnotetext{
*Author for correspondence. E-Mail: J.J.M.Braat@tnw.tudelft.nl
} 


$$
t(r, \varphi, z)=\exp (i l \varphi) \exp (i k z)
$$

implying a phase increase over one full turn of $l 2 \pi$ where $l$ is known as the topological charge, and $k=2 \pi \lambda$. We assume a wave propagating in the $z$ direction as $\exp [-i(\omega t-k z)]$. We have dropped the term $\exp (-i \omega t)$ since in the laboratory frame, the dynamics occur as the beam propagates through space not time. ${ }^{4}$

If $l$ is an integer, the orbital angular momentum has been increased by an amount of $l \hbar$. Of course, $l$ does not have to be limited to integer values; in that case, the expectation value for the angular momentum simply takes on the analog value of $l \hbar$. For the total beam, the angular momentum per unit area and per second is given by the quantity $l I / 2 \pi v$

with $I$ being the intensity of the beam and $v$ the frequency of the light. The wavefront with an $l=1$ topological charge is thus a helical function and is usually known as an optical vortex. Optical vortices may be formed using phase objects with a spiral phase depth. However, as Levenson et. al. ${ }^{5}$ have pointed out, it is not necessary to have a continuous phase structure to induce a topological charge. In Ref. 5 the authors used a quadrant structure with a full $\phi=2 \pi$ phase depth to form a vortex phase mask for optical lithography to print sub-wavelength features. A stepwise approximation to a spiral with a quadrant staircase structure (QSS) is sufficient (see Fig. 1). Kim et. al. ${ }^{6}$ have also shown that non-spiral phase plates are sufficient to induce a helical wavefront in a gaussian beam. In this work we shall use this approach to numerically model QSSs with dimension typically of $\sim 0.6 \lambda / N A$, to simulate the typical size of a structure on a recording surface. The change in depth $d$ (for the largest step) from the reference phase plane obeys, in reflection, the expression:

$$
d=\frac{3}{4} \cdot \frac{\phi \lambda}{4 \pi n_{0}}
$$

where $\phi=l \varphi(0 \leq \varphi<2 \pi)$ is the phase of the light and $n_{0}$ is the refractive index of the incident medium, usually air, so that $n_{0} \approx 1$. The factor $3 / 4$ arises from the fact that the step with the largest depth is the third of the four quadrants. The optical system is modeled using the Kirchhoff approximation and also the vectorial diffraction approach within DIFFRACT software ${ }^{7}$, in which a given amplitude distribution is Fourier-decomposed into plane waves and subsequently, the waves are superimposed after propagation in either the Fresnel or Fraunhofer regimes. ${ }^{8}$ We shall show that the vortex that exists within the background field gives rise to a rotation in the focal region of the emergent beam, as a direct result of the transfer of orbital angular momentum within the beam. It is this phenomenon that we shall exploit to propose a new method for multiplexing information from a single diffraction spot.

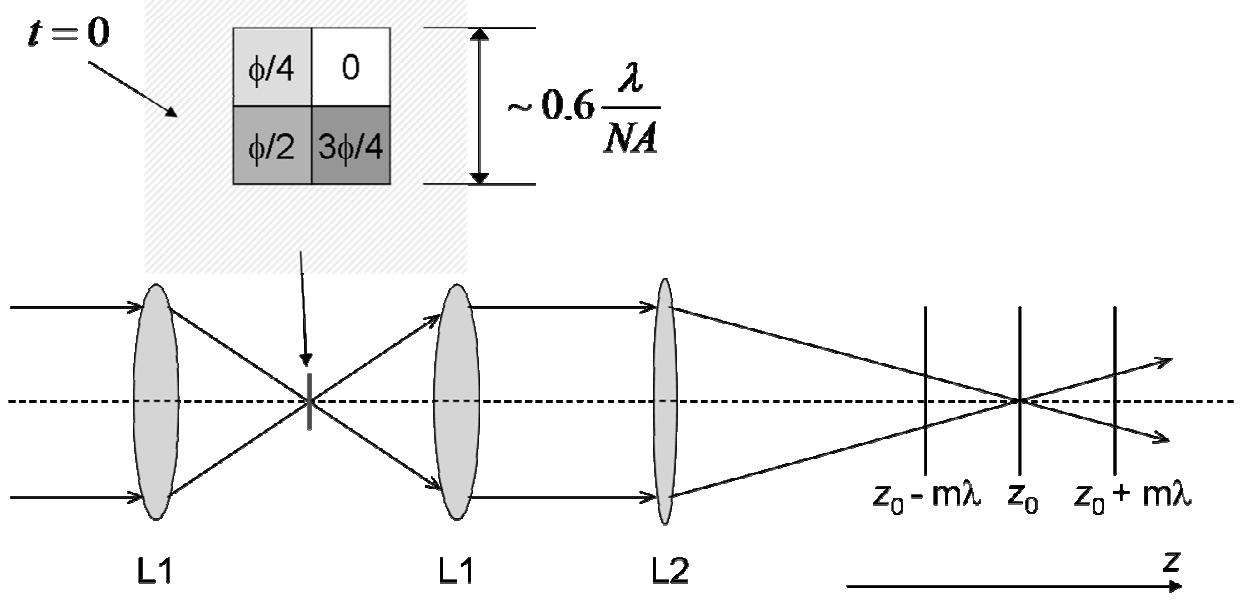

Fig. 1: A schematic of the simulated optical system. The incident Gaussian beam is focused onto a quadrant phase structure shown. The dimensions are of the order of a diffraction unit, and the transmissivity is unity. The region around the structure has a transmissivity of zero. The output beam is focused using L2 and we investigate the images of the vortex 25-45 wavelengths around the nominal focal point at $z_{0}$ (one focal depth amounts to approximately $8 \lambda$ in the simulated system). 
In this paper, for simplicity, we model isolated QSSs in an optical configuration equivalent to the one shown in Fig. 1. Although the configuration shows a transmissive mask, it is optically equivalent to a reflective phase object that would be encountered in reality. A plane polarized lowest order Gaussian light beam is focused down onto a QSS phase mask placed at the focal plane of two identical lenses L1 (of a given $N A$, typically 0.5 , and focal length $10000 \lambda$ ), and the emergent beam is focused by the lens L2 $(f=20000 \lambda, N A=0.25)$ and propagated in the $z$-direction to the focal region where the images are recorded typically $\pm \mathrm{m} \lambda$ each side of the focal plane at $z_{0}$ (see Fig. 1), where $\mathrm{m}$ is an integer typically 25-45 (note that the focal depth in this detection region approximately equals $8 \lambda$ ). The amplitude transmission through the four quadrants is set at unity. In our typical simulation the laser spot size was larger that the QSS dimension of $~ 0.6 N N A$, which gave rise to weak amplitude, specific to the wavefront helicity, at the focal region due to the large light intensity from outside of the quadrants. For this reason, we added an additional mask around the quadrants with $t=0$. It has been shown numerically that the rotation of optical vortices can be detected. ${ }^{9}$ Also, Arlt $^{10}$ has shown experimentally, that the characteristic rotation around the beam axis can be used to determine the handedness of the vortex. We propose to utilize this phenomenon of rotation within the Rayleigh range resulting in the variation of the intensity distribution to propose a new method for multiplexing information on an optical disk.

\section{RESULTS AND DISCUSSION}

We analyzed the intensity distributions of QSSs for phase depths ( $\phi$ ) of $2 \pi, 3 \pi / 2, \pi$ and $\pi / 2$ radians at the focal point at $z_{0}$. Figure 2 shows that there are significant differences in the spatial intensity variation. We find that a detection system e.g a quad-detector would yield significantly different voltage values for the various sum/difference combinations for the four phase depth values shown here. Having established that there is an intensity profile variation as a function of $\phi$, we proceeded to investigate the predicted field rotation in the focal region. These numerical results are presented next.
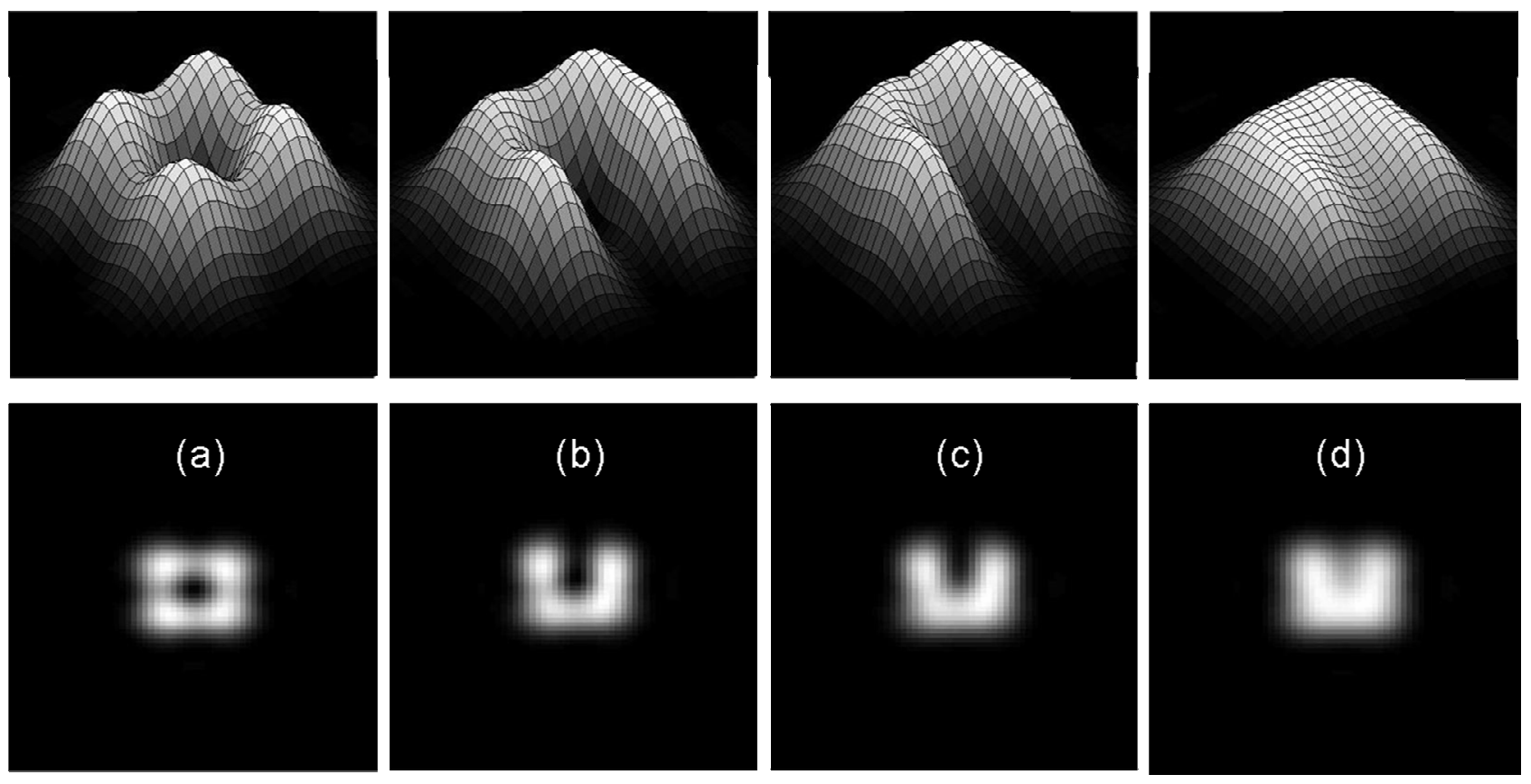

Fig. 2: $3 \mathrm{D}$ (top) and 2D (bottom) intensity distributions at the focal plane for QSS phase depths of (a) $2 \pi$, (b) $3 \pi / 2$, (c) $\pi$ and (d) $\pi / 2$ radians. As expected the $2 \pi$ phase depth shows clearly a phase singularity giving rise to zero intensity at the center. A detection system e.g. a quad detector at the focal point would yield differing values of intensity for the various sum/difference combinations. The vertical scale in the $3 \mathrm{D}$ plots is linear and in arbitrary units. The intensity profiles are approximately $6 \lambda$ in extent at the $1 / e^{2}$ level.

We shall now present numerical simulation data on the intensity distribution as a function of $z$ from $-40 \lambda$ to $+40 \lambda$ through the focal plane, for quadrant staircase structures with phase depths of $2 \pi, 3 \pi / 2, \pi$ and $\pi / 2$ radians. For a phase 
depth of $\phi=2 \pi$ - a full spiral, Fig. 3 shows how the images taken, in front of and behind the beam waist at $z_{0}$, change in intensity profile. A phase singularity in the case of the full $2 \pi$ staircase is located at the center of the image, and a rotation of the field intensity around a phase singularity can be clearly observed.
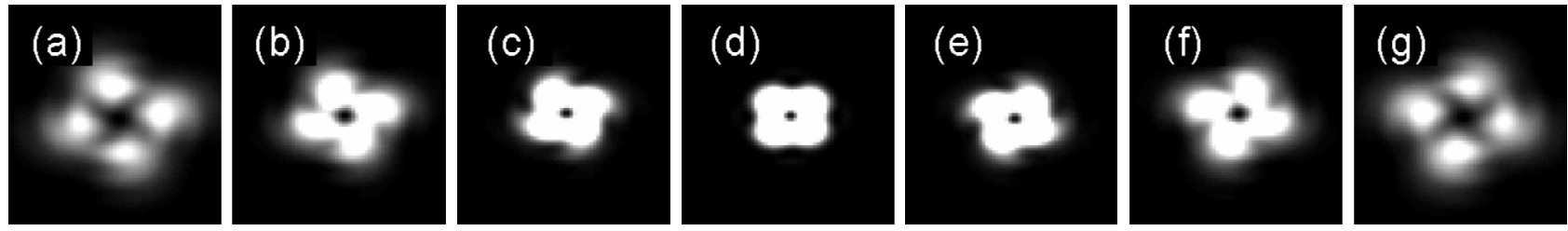

Fig. 3: Shows the rotation of the image in the focal region for a maximum phase depth of $\phi=2 \pi$. The images are taken at (a) $z_{0}-$ $45 \lambda$, (b) $z_{0}-30 \lambda$, (c) $z_{0}-15 \lambda$, (d) $z_{0}$, (e) $z_{0}+15 \lambda$, (f) $z_{0}+30 \lambda$, (g) $z_{0}+45 \lambda$. The sense (handedness) of the rotation turns out to be righthanded.
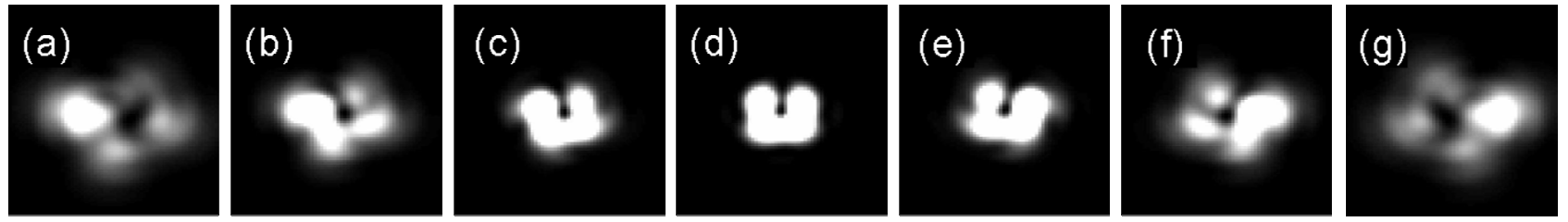

Fig. 4: Shows the rotation of the image in the focal region for a maximum phase depth of $\phi=3 \pi / 2$. The images are taken at (a) $z_{0}$ $45 \lambda$, (b) $z_{0}-30 \lambda$, (c) $z_{0}-15 \lambda$, (d) $z_{0}$ (focus), (e) $z_{0}+15 \lambda$, (f) $z_{0}+30 \lambda$, (g) $z_{0}+45 \lambda$. The sense of the rotation is again righthanded.
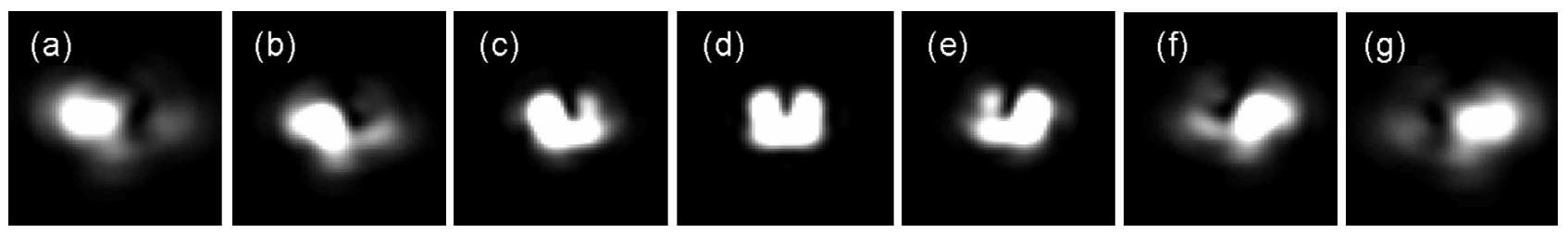

Fig. 5: Shows the rotation of the image in the focal region for a maximum phase depth of $\phi=\pi$. The images are taken at (a) $z_{0}-45 \lambda$, (b) $z_{0}-30 \lambda$, (c) $z_{0}-15 \lambda$, (d) $z_{0}$ (focus), (e) $z_{0}+15 \lambda$, (f) $z_{0}+30 \lambda$, (g) $z_{0}+45 \lambda$. The sense of the rotation is righthanded.

Similarly, in Fig. 4 we present data for a phase depth of $\phi=3 \pi / 2 \mathrm{rad}$. The intensity patterns and intensities are markedly different to those for $\phi=2 \pi \mathrm{rad}$ in Fig. 3. We have found such variation in $\phi=\pi$ (Fig. 5) and $\pi / 2 \mathrm{rad}$. We also found that the detail in the intensity distribution during rotation between $\pm 15 \lambda$ is more pronounced for lower values of $\phi$, e.g. $\phi<\pi$ rad. These data seem to suggest that by splitting the converging beam by means of e.g. a diffractive element, and placing quad-detectors or other multi-element detectors at varying distances from the focus effectively at $z_{0} \pm \mathrm{m} \lambda$, one should be able to distinguish between the various phase depths through the intensity distributions in the $z$ direction. Comparing figures 3-5 at each image plane, we deduce that multi-element detection would likely differentiate between structures with different phase depths. This leads to the possibility of multiple bits of information from a single area of $\sim 0.36(\lambda / N A)^{2} \mathrm{~nm}^{2}$.

In addition to the variation in the intensity distribution along the propagation $z$ direction, we have also investigated the effects of changing the order of the quadrants in the staircase, either by rotating the structure $90^{\circ}$ or $180^{\circ}$, or by 
changing the rotation sense. This is shown in Fig. 6, where, we obtain eight distinct images for a maximum phase depth of $\phi=\pi$ at the focal plane at $z_{0}$, four of which are shown in Fig. 6. This quadrant arrangement in Figs. 6 (a-d) gives rise to a lefthandedness of the field through the focal plane in the $z$-direction, as the wavefront propagates from $z_{0}-\mathrm{m} \lambda$ to $z_{0}+\mathrm{m} \lambda$. In addition, the image at the focal plane rotates, as expected, by the same angle as the QSS. The distinctiveness of each intensity profile coupled with a distinct rotation sense may yield multiple information from structures of the same phase depth $\phi$ but differing spatial intensity orientations.
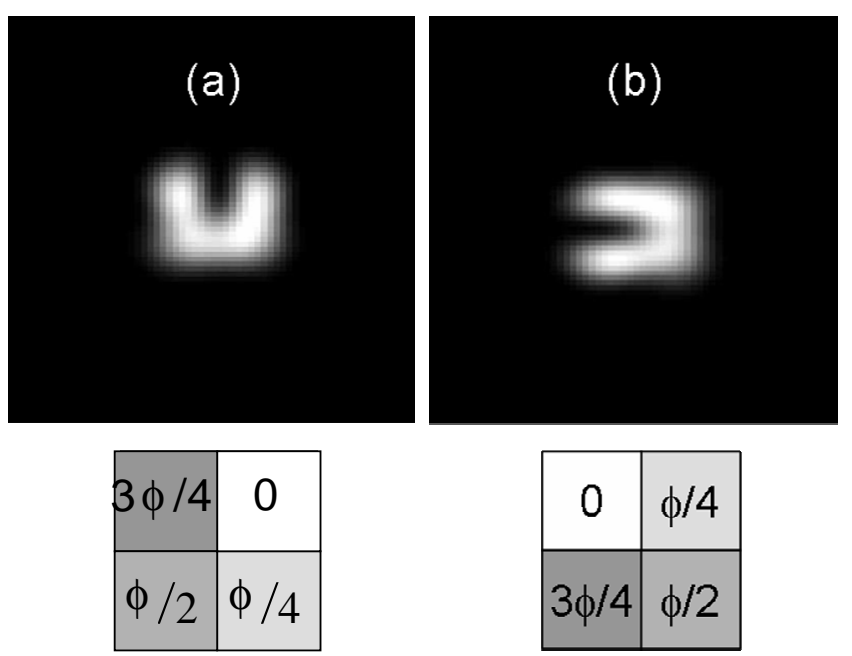
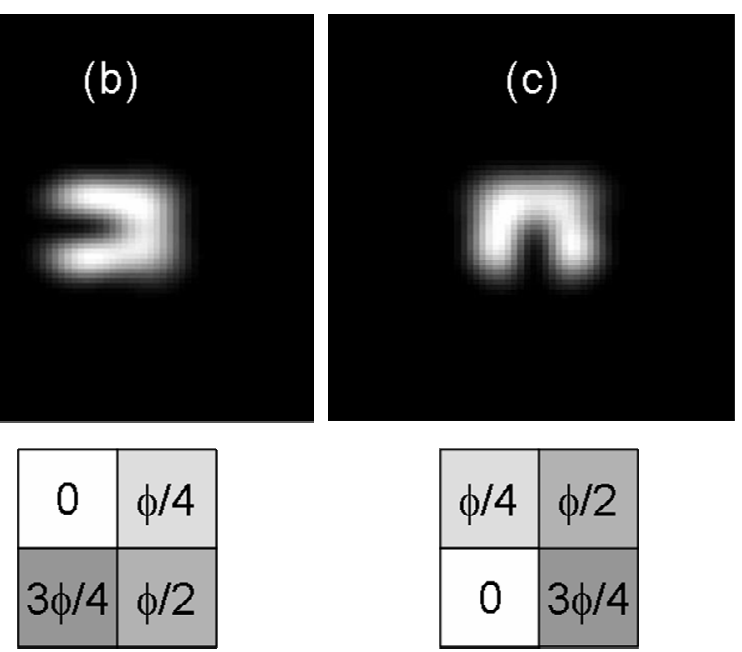
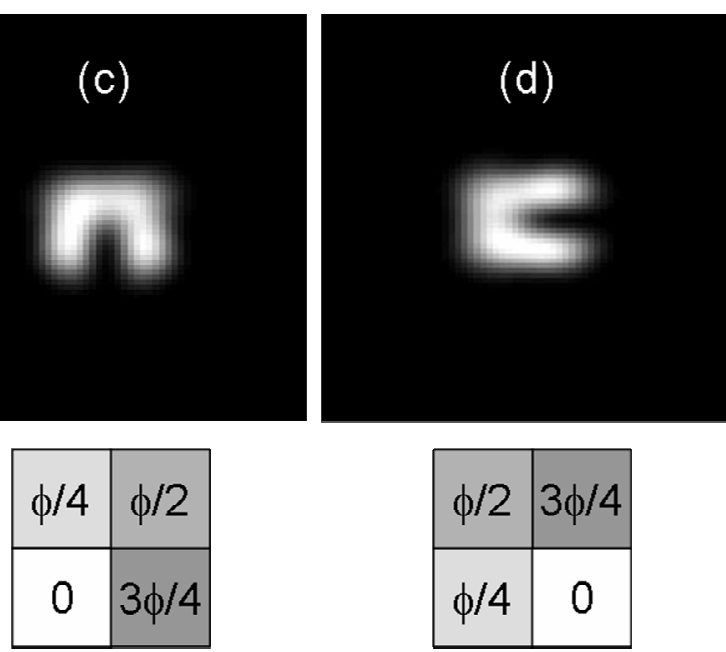

Fig. 6: Showing the quadrant orientation dependence of a $\phi=\pi$ phase depth structure on the field orientation at the focal plane at $z_{0}$. The quadrant arrangements in (a)-(d) give rise to a lefthanded rotation. Since the 'starting points' of the spatial intensity distribution are different, the combination of rotation sense and field distribution of a single phase depth may yield multiple and unique outputs.

\section{SUMMARY}

We have shown by numerical modeling that a staircase-like quadrant phase object may impart orbital angular momentum to a coherent Gaussian light beam. This angular momentum, due to its intrinsic conservation law is transferred along the beam, and manifests itself as a helical propagation of the Poynting vector which can be detected as a rotation in the focal region of an imaging lens. We have identified that due to a dependence of the intensity distribution on the longitudinal $z$ propagating direction, using multi-element detectors, e.g. quad-detectors, placed in the (equivalent) optical path along the effective $z$ direction (using e.g. a diffractive element), a multiplexed detection scheme may be realized. Work is underway to experimentally reproduce the numerical data. We have fabricated the quadrant staircase structures using photolithography and focused ion beam milling. The results of these experiments will be submitted for publication shortly.

\section{ACKNOWLEGEMENT}

This research was carried out in the framework of the European project IST-2000-26479 (SLAM).

\section{REFERENCES}

1. L. Allen, M.J. Padgett and M. Babiker, "The orbital angular momentum of light," in Progress in Optics XXXIX, E. Wolf, ed. (Elsevier Science, BV, 1999), pp. 291-372.

2. S.J. van Enk, "Geometric phase, transformations of gaussian light beams and angular momentum transfer," Opt. Commun., 102, 59-64 (1993). 
3. M.W. Beijersbergen, R.P.C. Coerwinkel, M. Kristensen and J.P. Woerdman, "Helical-wavefront laser beams produced with a spiral phaseplate," Opt. Commun., 112, 321-327 (1994).

4. D. Rozas, C.T. Law and G.A. Swartzlander, "Propagation dynamics of optical vortices," J. Opt. Soc. Am. B 14, 3054-3065 (1997).

5. M.D. Levenson, G. Dai and T. Ebihara, "The Vortex Mask: Making 80nm contacts with a twist!,” Proc. SPIE 4889, 1293-1303 (2002).

6. G.-H. Kim, J.-H. Jeon, K.-H. Ko, H.-J. Moon, J.-H. Lee and J.-S. Chang, "Optical vortices produced with a nonspiral phase plate," Appl. Opt., 36, 8614-8621 (1997).

7. DIFFRACT, MM Research Inc., Tucson, Arizona; http://www.mmresearch.com.

8. M. Mansuripur, "Certain computational aspects of vector diffraction problems," J. Opt. Soc. Am. A. 6, 786-805 (1989).

9. See for example, G.-H. Kim, H.-J. Lee, J.-U. Kim and H. Suk, "Propagation dynamics of optical vortices with anisotropic phase profiles," J. Opt. Soc. Am. B 20, 351-359 (2003).

10. J. Arlt, "Handedness and azimuthal energy flow of optical vortex beams," J. Mod. Opt., 50, 1573-1580 (2003). 